\title{
Modelo de crecimiento microbiano para predecir el comportamiento de Salmonella spp. en queso costeño colombiano
}

Rafael E. González ${ }^{\star}$, Arnulfo Tarón Dunoyer² y Jaime Pérez Mendoza1.

(1) Facultad de Ingeniería, Dpto. de Ciencia de los Alimentos, Universidad de Cartagena, Piedra de Bolívar Av. Del Consulado, Calle 30 N 48-157, Cartagena. Colombia. Correo-e: rgonzalezc1@unicartagena.edu.co; jperezm@unicartagena.edu.co

(2) Facultad de Ingeniería, Dpto. de Operaciones Unitarias, Universidad de Cartagena, Piedra de Bolívar

Av. Del Consulado, Calle 30 № 48-157Cartagena. Colombia. Correo-e: atarond@unicartagena.edu.co

* Autor a quien debe ser dirigida la correspondencia

Recibido Jun. 29, 2021; Aceptado Sep. 1, 2021; Versión final Oct. 13, 2021, Publicado Feb. 2022

\section{Resumen}

El objetivo de este estudio fue desarrollar y validar un modelo secundario para predecir el crecimiento de la bacteria patógena Salmonella spp. en queso costeño de Colombia. Muestras de queso fueron inoculadas con Salmonella spp. y almacenadas a distintas temperaturas $\left(8,14,18\right.$ y $\left.21^{\circ} \mathrm{C}\right)$. El modelo Baranyi y Roberts fue utilizado para obtener los parámetros cinéticos como la velocidad especifica de crecimiento $\left(\mu_{\max }\right)$. Los valores de $\mu_{\max }$ se emplearon para construir el modelo secundario a partir de una ecuación polinómica. El proceso de validación mostró valores de factor de exactitud (Af) de 1,112 y sesgo (Bf) de 0,898 y bajos valores $(0,001)$ de error cuadrático medio (ECM), confirmando que el modelo puede predecir el crecimiento de Salmonella spp. en este tipo de matriz alimentaria. En conclusión, el modelo propuesto puede ser aplicado por los productores para optimizar la calidad del queso costeño colombiano, evitando posibles intoxicaciones a causa del crecimiento de esta bacteria patógena.

\section{Microbial growth model to predict growth behavior of Salmonella spp. on Colombian coastal cheese}

\begin{abstract}
The objective of this study was to develop and validate a secondary model to predict the growth of the pathogenic bacteria Salmonella spp. on Colombian coastal cheese. Cheese samples were inoculated with Salmonella spp. and stored at different temperatures $\left(8,14,18\right.$, and $\left.21^{\circ} \mathrm{C}\right)$. The Baranyi and Roberts model was applied to obtain kinetic parameters, including specific growth rates $\left(\mu_{\max }\right)$, which served to build a secondary model from a polynomial equation. The validation process showed an accuracy factor (Af) of 1.112, a bias (Bf) of 0.898 , and a low (0.001) mean square error (MSE). This confirmed that the model accurately predicted the growth of Salmonella spp. on this type of food matrix. In conclusion, the proposed model can be applied by producers to optimize the quality of Colombian coastal cheese while avoiding possible food poisoning events caused by growth of Salmonella pathogenic bacteria.
\end{abstract}




\section{INTRODUCCIÓN}

Los productos lácteos son susceptibles de contaminación microbiológica debido a su composición nutricional $\mathrm{pH}$ y contenido de humedad. El queso costeño es un producto listo para consumo después de su elaboración, además, es clasificado según su consistencia y humedad en: extraduro, duro, firme y blando. Es producido y distribuido originalmente en la región en la región Caribe colombiana, en los departamentos de Magdalena, Atlántico, Córdoba, Sucre, Bolívar, La Guajira y Cesar, aunque también su consumo se ha extendido a otros departamentos como Cundinamarca, Santander y Antioquia, convirtiéndose en un renglón importante del desarrollo económico del sector agropecuario. Normalmente es un producto elaborado a través de prácticas artesanales a partir de leche de vaca no pasteurizada mediante coagulación enzimática o acidificación, pero sin la adición de cultivos iniciadores (Gutiérrez et al., 2017). El proceso de elaboración de esta matriz alimentaria no se encuentra estandarizado incluso su almacenamiento no es adecuado respecto a las condiciones higiénicas, temperatura, humedad, etc. (Oliver et al., 2005). El queso, es reconocido como uno de los productos lácteos más comercializados en la región del Caribe colombiano. Las características físicoquímicas del queso lo hacen un sustrato conveniente para el crecimiento de microorganismos de origen alimentario incluyendo enterobacterias como Salmonella spp (Tirloni et al., 2021).

Salmonella spp es uno de los patógenos transmitidos por alimentos más comunes en animales y humanos, más de 2600 serotipos han sido reportados basándose en el antígeno de superficie (Kipper et al., 2019). Las infecciones ocasionadas por este patógeno ocasionan dolor abdominal, diarrea, gastroenteritis y fiebre. Según las estimaciones de la Organización Mundial de la Salud (OMS), aproximadamente 1.300 millones de personas contraen gastroenteritis o diarrea, y más de 3 millones de personas mueren debido a Infección por Salmonella spp (Sannigrahi et al., 2020), Salmonella spp afecta la salud de las personas y la seguridad alimentaria más que otros patógenos transmitidos por los alimentos (Herrero y Olsen, 2018).

La microbiología predictiva se ha convertido en una herramienta importante que respalda la seguridad alimentaria en los sistemas de gestión. El uso de modelos predictivos por parte de los productores de alimentos ha incrementado continuamente con el paso del tiempo debido a las mejoras en la calidad final de los productos elaborados. Un modelo es una descripción simplificada de las relaciones entre las observaciones del sistema y los factores ambientales que causan la respuesta microbiana. Por tanto, los modelos predictivos se utilizan para evaluar riesgos, así como la determinación de los puntos críticos de control en el Análisis de Peligros y Puntos Críticos de Control (APPCC) mediante la estimación de cambios en el número de microorganismos en la cadena de producción. La microbiología predictiva es un área multidisciplinar, donde trabajan juntos estadísticos, microbiólogos de alimentos, matemáticos e informáticos.

En términos generales, se pueden construir modelos predictivos con el fin de mejorar la calidad de los alimentos, ya que estos modelos permiten anticiparse al comportamiento de los microorganismos en determinadas matrices alimentarias y tomar así las acciones correctivas pertinentes. El desarrollo de modelos predictivos de crecimiento bacteriano es una herramienta importante para la industria de los alimentos (Tarlak et al., 2020); ya que a través de ellos se puede evaluar el riesgo de crecimiento de bacterias patógenas para prevenir intoxicaciones alimentarias. (Lee et al., 2014; Milkievicz et al., 2020). Los modelos predictivos han sido utilizados con el fin de proporcionar predicciones confiables del crecimiento microbiano en productos alimenticios bajo condiciones ambientales cambiantes de temperatura, $\mathrm{pH}$, actividad de agua y presencia de sustancias químicas (Jung y Schaffner, 2021). Con el fin de evaluar el desempeño y calidad del ajuste del modelo se han propuesto diferentes índices estadísticos como: error cuadrático medio (ECM), factor de exactitud (Af) y factor de sesgo (Bf), (Park et al., 2020).

En microbiología predictiva, las expresiones matemáticas utilizadas para describir el comportamiento de los microorganismos se clasifican en modelos primarios, secundarios y terciarios. Los modelos primarios refieren la evolución en concentraciones bacterianas en función al tiempo basados en los parámetros cinéticos: velocidad especifica de crecimiento $\left(\mu_{\max }\right)$, fase de latencia $(\lambda)$ y concentración final de bacterias $\left(Y_{\max }\right)$. Estos parámetros se pueden obtener al ajustar un modelo primario a los datos experimentales de crecimiento. Los modelos secundarios se enfocan en las respuestas de los parámetros de crecimiento producidas por las fluctuaciones en las condiciones ambientales (Whiting, 1995). Los modelos terciarios son modelos que usan los parámetros cinéticos estimados con los modelos secundarios para ser capaces de predecir la evolución de concentraciones bacterianas en función de las condiciones ambientales. Varios modelos como el de Baranyi, Gompertz y logístico han sido utilizados para predecir el crecimiento microbiano, por ejemplo, el modelo Baranyi se ajusta de mejor manera en predecir el efecto de la temperatura sobre el crecimiento de Salmonella spp en pollo (Juneja et al., 2007).

Existen modelos capaces de predecir el comportamiento de Salmonella spp en diferentes matrices alimentarias como salami (Orosco et al., 2021), vegetales (Jung y Schaffner, 2021), pollo (Milkievicz et al., 2021) queso ricotta (Tirloni et al., 2019). Sin embargo, hasta el momento no se encuentran reportes en la literatura sobre modelos que permitan predecir el comportamiento de Salmonella spp en queso costeño 
colombiano. Por tanto, el objetivo del presente estudio fue desarrollar y validar un modelo predictivo sobre el comportamiento de Salmonella spp en queso costeño colombiano.

\section{MATERIALES Y MÉTODOS}

Para las condiciones de activación, inoculación y enumeración de Salmonella spp, determinación de valores de $\mathrm{pH}$ y contenido de agua en queso costeño, así como el modelamiento del comportamiento de Salmonella spp a través de Baranyi y Roberts y la validación del modelo polinómico desarrollado se usaron los siguientes materiales y métodos

\section{Condiciones de crecimiento bacteriano}

Salmonella spp fue obtenida del laboratorio de microbiología de alimentos de la Universidad de Cartagena (Colombia). Inicialmente el microorganismo patógeno fue transferido a tubos con caldo Brain Heart Infusion (BHI) (Becton Dickinson $\mathrm{GmbH}$, Alemania) e incubado a $37^{\circ} \mathrm{C}$ durante $24 \mathrm{~h}$ en una incubadora (memmert IN 160). Posteriormente los cultivos fueron re -incubados en tubos conteniendo $\mathrm{BHI}$ a diferentes temperaturas $\left(8,14,18\right.$ y $\left.21^{\circ} \mathrm{C}\right)$ con el fin de adaptar las células a las diferentes condiciones ambientales y lograr una concentración bacteriana de 2 Log UFC/mL; la cual fue obtenida después de realizar diluciones seriadas consecutivas utilizando solución salina $(0,85 \%$ p/v) esteril y confirmada mediante recuento en placa.

\section{Inoculación y enumeración de Salmonella spp}

Las muestras de queso fueron obtenidas en un local comercial (Cartagena D.T y C., Colombia) el mismo día de la producción. Un total de $5 \mathrm{Kg}$ de queso fueron cortadas bajo condiciones de asepsia en porciones de $11 \mathrm{~g}$ cada una utilizando cuchillos estériles. Las muestras fueron transportadas en bolsas estériles dentro de una cuba conteniendo en hielo seco, y una vez en el laboratorio fueron mantenidas a $4^{\circ} \mathrm{C}$ e inoculadas dentro de las $24 \mathrm{~h}$ siguientes, con el fin de minimizar cambios en las propiedades fisicoquímicas. Antes de llevar a cabo las cinéticas de crecimiento, se realizaron análisis microbiológicos en búsqueda de Salmonella spp en las muestras de queso. Luego, de corroborar la ausencia del patógeno en las muestras, $100 \mu \mathrm{L}$ de la suspensión de Salmonella spp fueron adicionados a la superficie de cada muestra de $11 \mathrm{~g}$ de queso (20 muestras aproximadamente). Después, las muestras fueron ubicadas en bolsas estériles y almacenadas a diferentes temperaturas $\left(8,14,18\right.$ y $21^{\circ} \mathrm{C}$ ) (simulando temperaturas de refrigeración y de abuso de almacenamiento) para construir las curvas de crecimiento microbiano a cada temperatura. Posteriormente, las muestras fueron mezcladas con $99 \mathrm{~mL}$ de agua de peptona tamponada e incubadas durante $24 \mathrm{~h}$, luego se realizó un enriquecimiento selectivo en caldo tetrationato (Laboratorios Britania, Argentina) por 24 h. F, a continuación, se llevó a cabo el aislamiento en medios BD Hektoen y Xylose-Lysine-Desoxycholate (XLD), (Becton Dickinson $\mathrm{GmbH}$, Alemania) para finalmente realizar confirmación bioquímica del microorganismo. Es importante destacar que el conteo de Salmonella spp fue llevado a cabo por siembra en superficie en agar XLD por triplicado y las cajas de Petri fueron incubadas (memmert IN 160) a $37^{\circ} \mathrm{C}$ durante $24 \mathrm{~h}$. Los resultados fueron expresados en Log UFC/g.

\section{Estudio analítico del queso}

Las determinaciones de Aw fue llevada a cabo utilizando un AquaLab 4 TE (METER Group, Inc. Pullman, Washington, USA), mientras que las mediciones de $\mathrm{pH}$ con un potenciómetro digital (Beckman, USA). Todos los análisis fueron realizados por triplicado al inicio y al final de cada cinética de crecimiento $\mathrm{s}$

\section{Modelado primario}

Los datos experimentales de las cinéticas de crecimiento de Salmonella spp en queso costeño fueron ajustados al modelo Baranyi y Roberts (1994) (Ecuación 1).

$$
(\mathrm{t})=\mathrm{y}_{0}+\mu_{\max } \mathrm{t}+\frac{1}{\mu_{\max }} \ln \left(\mathrm{e}^{-\mathrm{vt}}+\mathrm{e}^{-\mathrm{h}_{0}}-\mathrm{e}^{-\mathrm{vt}-\mathrm{h}_{0}}\right)-\frac{1}{\mathrm{~m}} \ln \left[1+\frac{\mathrm{e}^{\mathrm{m} \mu_{\max } \mathrm{t}+\frac{1}{\mu_{\max }} \ln \left(\mathrm{e}^{-v \mathrm{t}}+\mathrm{e}^{\left.-\mathrm{h}_{0}-\mathrm{e}^{-v \mathrm{t}-\mathrm{h}_{0}}\right)}-1\right.}}{\mathrm{e}^{\mathrm{m}\left(\mathrm{y}_{\max }-\mathrm{y}_{0}\right)}}\right]
$$

Donde y $(\mathrm{t})$ es la concentración celular o el diámetro de las colonias, yo es la concentración o diámetro inicial, $\mu_{\text {máx }}$ es la velocidad específica de crecimiento $(1 / \mathrm{h}), \mathrm{m}$ es un parámetro de curvatura para caracterizar la transición de la fase exponencial, $v$ es un parámetro de curvatura para caracterizar la transición a la fase exponencial y ho es un parámetro adimensional que cuantifica el estado fisiológico inicial de las células. 


\section{Modelamiento secundario}

Para llevar a cabo este modelamiento se utilizó una ecuación polinómica utilizando la temperatura y la velocidad de crecimiento. La ecuación polinómica fue la siguiente:

$\operatorname{In}(x)=a+b * T+c * T^{2}$

Donde, In $x$ es el logaritmo natural de la velocidad de crecimiento $a, b$, and $c$ son constantes y $T$ es la temperatura.

\section{Validación del modelo predictivo}

El factor de exactitud (Af), el factor de sesgo (Bf), y el error cuadrático medio (ECM) fueron utilizados para evaluar el desempeño del modelo predictivo desarrollado (Jung y Schaffner, 2021). Las ecuaciones de Af, Bf y ECM son las siguientes:

$$
\begin{aligned}
& A f=10^{\left(\left.\sum\right|^{\log \mu \text { pred }} / \log \mu \text { obs } \mid / n\right)} \\
& B f=10^{\left(\sum \log \left(\frac{\text { o obs }}{\mu \text { pred }}\right) / n\right)} \\
& E C M=\frac{\sum(\text { obs }- \text { pred })^{2}}{n}
\end{aligned}
$$

Donde los factores obs, pred son los valores observados y predichos de $\mu_{\max }$; mientras que $\mathrm{n}$ corresponde al número de repeticiones de los datos observados.

\section{Análisis estadístico}

Los parámetros de crecimiento de Salmonella spp y las determinaciones analíticas son expresados como media aritmética y desviación estándar. La influencia de la temperatura sobre los factores de crecimiento, $\mathrm{pH}$ y Aw fueron determinadas mediante un análisis normal de varianza de un factor utilizando la prueba de tukey con un nivel de significancia del 95\%. mediante el programa de cómputo SPSS (Statistical Package for the Social Sciences) versión 17.0 para Windows (SPSS Inc. Chicago, IL, USA).

\section{RESULTADOS Y DISCUSIÓN}

Los resultados se presentan en 3 subsecciones: i) Ajuste de los datos de crecimiento de Salmonella spp al modelo Baranyi y Roberts; ii) Determinaciones analíticas, iii) Modelo secundario.

Ajuste de los datos de crecimientos de Salmonella spp.

Salmonella spp no fue detectado en las muestras de queso utilizadas como sustrato. La conveniencia de los modelos utilizados para el desarrollo de modelos predictivos está basada principalmente en factores como el tipo de microorganismo, las matrices alimentarias usadas como sustrato y las condiciones ambientales en las cuales fue desarrollado el modelo. En el presente estudio, el modelo de Baranyi y Roberts (1994) fue ajustado a los datos de las cinéticas de crecimiento de Salmonella spp en queso costeño almacenado a distintas temperaturas $\left(8,14,18\right.$ y $\left.21^{\circ} \mathrm{C}\right)$. En la figura 1 se aprecian las curvas de crecimiento de Salmonella spp, en donde se puede apreciar una concentración inicial de Salmonella spp cercana a 2 Log UFC/g para todas las temperaturas evaluadas, así como un incremento variado en la población de Salmonella spp. Estos resultados muestran que el crecimiento de este patógeno en queso costeño, es afectado por los cambios de temperatura. Es importante destacar que la utilización de los modelos primarios como el de Baranyi y Roberts permite calcular parámetros de crecimiento del microorganismo: conteo inicial de células $\left(\mathrm{Y}_{0}\right)$, velocidad de crecimiento $\left(\mu_{\max }\right)$ y concentración final de células $\left(Y_{\max }\right)$, las cuales son empleadas para desarrollar modelos secundarios.

Los valores sobre los parámetros de crecimiento de Salmonella spp en queso costeño se muestran en la tabla 1 , en donde se observa que $Y_{0}$ no cambio significativamente $(p>0,05)$ por efecto de la temperatura, indicando que $Y_{0}$ depende mayoritariamente de la concentración del microorganismo adicionada en la matriz alimentaria. Los valores de $Y_{0}$ estuvieron entre 1,844 y 1,977 Log UFC/g; los cuales corresponden a las concentraciones adicionadas del microorganismo patógeno a las muestras de queso, lo cual corrobora la ausencia del patógeno en las muestras de queso antes de llevar a cabo las cinéticas de crecimiento. El mayor valor de $Y_{0}$ fue obtenido a $21{ }^{\circ} \mathrm{C}$ con 1,977 UFC/g; mientras que el menor valor (1,844 log UFC/g) se logró a una 
temperatura de $14^{\circ} \mathrm{C}$. El número inicial de una bacteria patógena es un indicador crucial de seguridad microbiológica en productos lácteos artesanales, especialmente en quesos jóvenes (Acai et al., 2014). Los valores de $Y_{0}$ adquieren mayor importancia en el desarrollo de productos fermentados, ya que puede servir para optimizar la concentración de bacterias probióticas a inocular en la materia prima. Además, $Y_{0}$ no ha sido un parámetro tradicionalmente usado para el desarrollo de modelos secundarios, lo cual puede ser atribuido a la poca variabilidad o a que puede ser manipulado fácilmente mediante el control del número de bacterias incorporadas en la matriz alimentaria.

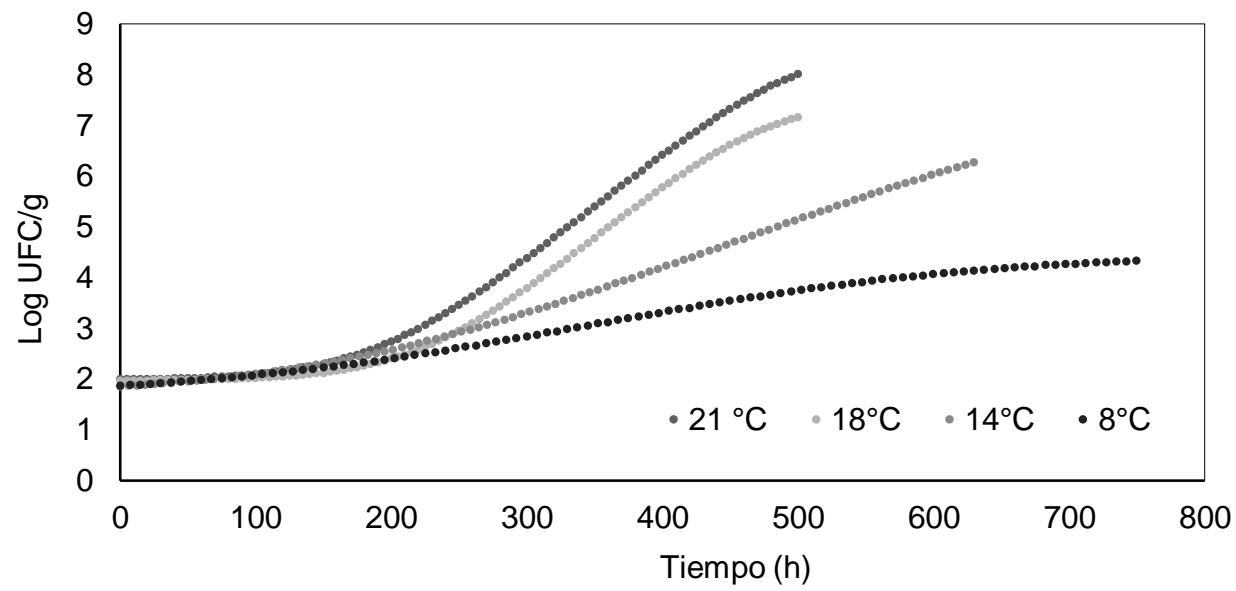

Fig. 1: Curvas de crecimiento de Salmonella spp utilizando el modelo Baranyi y Roberts

Con respecto a los valores de $\mu_{\max }$, se puede inferir que tuvo una relación proporcional con los incrementos de temperatura. Es decir, $\mu_{\max }$ incrementó al aumentar la temperatura. El valor mayor de $\mu$ fue obtenido a $21^{\circ} \mathrm{C}\left(0,027 \mathrm{~h}^{-1}\right)$ seguido por las muestras de queso almacenadas a $18^{\circ} \mathrm{C}\left(0,023 \mathrm{~h}^{-1}\right)$ y $12^{\circ} \mathrm{C}\left(0,010 \mathrm{~h}^{-1}\right)$; mientras que el menor valor fue alcanzado a $8^{\circ} \mathrm{C}\left(0,008 \mathrm{~h}^{-1}\right)$. Estos valores fueron menores a los publicados por Tirloni et al., (2021) quienes encontraron valores entre 0,785 y 0,847 $\mathrm{h}^{-1}$ en Salmonella spp creciendo en queso ricotta, esta discrepancia en los valores de $\mu$ puede ser consecuencia de las diferencias en la composición nutricional de los quesos y el tipo de manufactura; ya que el queso costeño no es sometido a un proceso de pasteurización y pueden encontrarse presentes microorganismos que compitan por los nutrientes. Por otro lado, entendiendo el abuso de temperatura como temperaturas superiores a la refrigeración, se podría decir que este abuso facilita el crecimiento de Salmonella spp en un producto típico de la región caribe colombiana como el queso costeño. Algunos autores han reportado que $\mu$ es principalmente afectado por las limitaciones de nutrientes, oxígeno y la producción de metabolitos que puedan ocasionar un incremento del estrés medio ambiental (Skandamis y Jeanson, 2015).

Tabla 1: Parámetros de crecimiento de Salmonella spp utilizando el modelo Baranyi y Roberts. Filas de mismo análisis sin ninguna letra en común presentaron diferencias significativas a un nivel $p<0,05$

\begin{tabular}{|c|c|c|c|c|c|}
\hline Temperatura $\left({ }^{\circ} \mathrm{C}\right)$ & $Y_{0}(\log$ UFC/g) & $\mu_{\max }\left(h^{-1}\right)$ & $Y_{\max }(\log$ UFC/g) & $h_{0}$ & $R^{2}$ \\
\hline 21 & $1,977 \pm 0,02^{\mathrm{a}}$ & $0,027 \pm 0,01^{\underline{a}}$ & $8,700 \pm 0,21^{\mathrm{a}}$ & $4,273 \pm 0,00^{\mathrm{a}}$ & 0,987 \\
\hline 18 & $1,961 \pm 0,01^{\mathrm{a}}$ & $0,023 \pm 0,00^{\mathrm{a}}$ & $7,586 \pm 0,18^{\mathrm{b}}$ & $5,181 \pm 0,03^{\mathrm{b}}$ & 0,958 \\
\hline 14 & $1,844 \pm 0,01^{\mathrm{a}}$ & $0,010 \pm 0,01^{\mathrm{b}}$ & $7,616 \pm 0,14^{\mathrm{b}}$ & $1,910 \pm 0,11^{\mathrm{c}}$ & 0,998 \\
\hline 8 & $1,855 \pm 0,01^{\mathrm{a}}$ & $0,008 \pm 0,00^{\mathrm{b}}$ & $4,466 \pm 0,12^{\mathrm{c}}$ & $1,557 \pm 0,04^{\mathrm{c}}$ & 0,994 \\
\hline
\end{tabular}

$Y_{\max }$ corresponde a la máxima concentración bacteriana alcanzada por Salmonella spp al final de la fase logarítmica. Este parámetro reviste especial relevancia, ya que algunas bacterias producen toxinas cuando alcanzan un cierto número de microorganismos. Los cambios de temperatura, como era previsible afectaron significativamente los valores de $Y_{\max }$. El mayor valor para $Y_{\max }$ fue obtenido a $21^{\circ} \mathrm{C}$ con $8,700 \mathrm{UFC} / \mathrm{g}$; mientras que el menor valor se obtuvo a $8^{\circ} \mathrm{C}$ de almacenamiento con $4,466 \mathrm{UFC} / \mathrm{g}$, en las restantes temperaturas se obtuvieron valores intermedios; es decir, $7,586 \mathrm{UFC} / \mathrm{g}$ a $18^{\circ} \mathrm{C}$ y $7,616 \mathrm{UFC} / \mathrm{g}$ a $14^{\circ} \mathrm{C}$. Aunque, en líneas generales, los resultados obtenidos soportan la premisa que las temperaturas bajas como es evidente, resultan mejores en la conservación de queso costeño, ya que disminuyen la velocidad de crecimiento de Salmonella spp y reducen el número final de bacterias presentes en el queso, con lo cual se disminuyen las posibilidades de producción de toxinas exógenas; el real objetivo de este trabajo es obtener un modelo secundario de fácil utilización para predecir el comportamiento de una bacteria patógena como Salmonella spp en queso costeño, ya que hasta el momento no existen estudios sobre esta temática. El último parámetro 
calculado por el modelo de Baranyi y Roberts fue ho que corresponde al estado fisiológico del microorganismo. Este parámetro presentó una variación significativa atribuida a los cambios llevados a cabo a fin de mejorar la calidad del ajuste primario en los datos iniciales de crecimiento de Salmonella spp.

\section{Determinaciones analíticas}

Teniendo en cuenta que el queso costeño es un producto alimenticio elaborado de manera artesanal mayoritariamente en Colombia, que la presencia de bacterias autóctonas y en particular ácido lácticas pueden producir compuestos con características antimicrobianas y que las modificaciones en los valores de $\mathrm{pH}$ y $\mathrm{Aw}$ pueden influenciar el crecimiento de microorganismos patógenos (Leong et al., 2014). Es relevante verificar que el queso no tenga una presencia previa de Salmonella spp como efectivamente se realizó en este trabajo. Con respecto a las determinaciones analíticas llevadas a cabo, como se puede apreciar en la tabla 2, los valores de Aw determinados en el inicio de la cinética de crecimiento no fueron modificados significativamente $(p>0,05)$ por efecto de la temperatura, dichos valores de Aw se encontraron dentro del rango 0,982 - 0,964. Sin embargo, cuando se comparan estos valores con los conseguidos al final de las cinéticas de crecimiento, se presentan diferencias significativas $(p<0,05)$, las cuales se acentuaron al incrementar la temperatura. Finalizando las cinéticas de crecimiento, los mayores valores de Aw 0,952 y 0,941 fueron obtenidos a 14 y $8^{\circ} \mathrm{C}$ respectivamente, mientras que valores menores se lograron a 21 y $18{ }^{\circ} \mathrm{C}$ con 0,880 y 0,890 respectivamente. Para resumir, el Aw es mayor al iniciar las cinéticas de crecimiento de Salmonella spp, en comparación con los valores de Aw encontrados al término de las cinéticas, tal comportamiento puede ser atribuido tanto al crecimiento de la bacteria, ya que los mayores valores de $\mu$ también fueron logrados a 21 y $18^{\circ} \mathrm{C}$, aunque también se debe considerar la sinéresis que experimenta el queso. Resultados similares fueron reportados por Tirloni et al., (2021) quienes encontraron valores de Aw entre 0,996 y 0,999 en quesos ricota de distintas marcas comerciales antes de inocular Salmonella spp y desarrollar un modelo matemático para describir el comportamiento de esta bacteria. Por el contrario, estos autores, no determinaron el efecto de la temperatura sobre el Aw y tampoco reportaron encontraron diferencias significativas $(p>0,05)$ en cuanto a $\mathrm{pH}$, humedad, ácido láctico, acético y cítrico y concentraciones de sales entre las distintas marcas de queso evaluadas.

En relación a los valores de $\mathrm{pH}$ al empezar la cinética, se pudo notar un menor valor $(6,24) \mathrm{a} 21^{\circ} \mathrm{C}$, mientras que el mayor valor fue 6,34 conseguido a $8^{\circ} \mathrm{C}$, lo que representa una baja influencia de la temperatura en la producción de ácido al iniciar la cinética. Valores intermedios fueron obtenidos a 18 y $14^{\circ} \mathrm{C}$ con 6,21 y 6,33 respectivamente. En cuanto a los valores de $\mathrm{pH}$ encontrados al finalizar las cinéticas de crecimiento, nuevamente el mayor valor fue $6,31\left(8^{\circ} \mathrm{C}\right)$ y el menor valor $6,14\left(21^{\circ} \mathrm{C}\right)$; es decir no hay una producción significativa de compuestos que modifiquen los valores de $\mathrm{pH}$ durante el tiempo que duran las respectivas cinéticas de crecimiento, este comportamiento corrobora la débil producción de estos compuestos por parte de Salmonella spp. Los resultados de $\mathrm{pH}$ reportados en el presente trabajo son similares a los publicados por Tirloni et al., (2021) quienes encontraron valores para queso ricota de distintas marcas comerciales entre 5,96 y 6,62 al momento de la inoculación de Salmonella spp, estos autores no determinaron los análisis fisicoquímicos al finalizar las cinéticas.

Tabla 2: Valores de $\mathrm{pH}$ y Aw de las muestras del queso al inicio y al final de las cinéticas de crecimiento. Columnas y filas de mismo análisis sin ninguna letra en común presentaron diferencias significativas a un nivel $p<0,05$

\begin{tabular}{|c|c|c|c|c|}
\hline Temperatura $\left({ }^{\circ} \mathrm{C}\right)$ & $A w$ (inicial) & $A w$ (final) & $p H$ (inicial) & $p H$ (final) \\
\hline 21 & $0,964 \pm 0,02^{\underline{a}}$ & $0,880 \pm 0,13^{\mathrm{b}}$ & $6,24 \pm 0,00^{\mathrm{a}}$ & $6,14 \pm 0,00^{\mathrm{b}}$ \\
\hline 18 & $0,976 \pm 0,09^{\mathrm{a}}$ & $0,890 \pm 0,03^{\mathrm{b}}$ & $6,21 \pm 0,00^{\mathrm{a}}$ & $6,20 \pm 0,05^{\mathrm{a}}$ \\
\hline 14 & $0,982 \pm 0,13^{\mathrm{a}}$ & $0,952 \pm 0,00^{\mathrm{a}}$ & $6,33 \pm 0,05^{\mathrm{a}}$ & $6,30 \pm 0,05^{\mathrm{a}}$ \\
\hline 8 & $0,982 \pm 0,20^{\mathrm{a}}$ & $0,941 \pm 0,01^{\mathrm{a}}$ & $6,34 \pm 0,00^{\mathrm{a}}$ & $6,31 \pm 0,00^{\mathrm{a}^{\mathrm{a}}}$ \\
\hline
\end{tabular}

\section{Modelo secundario}

En este estudio, se simuló un evento de contaminación de manera controla al incorporar 2,0 UFC/g de Salmonella spp en queso costeño con el fin de obtener los datos para construir el modelo secundario, ya que generalmente los modelos predictivos disponibles son desarrollados utilizando medios de cultivo comerciales en lugar de matrices alimentarias y por ende presentan inconvenientes al momento de realizar las predicciones. Para la construcción del modelo secundario fueron utilizados los valores de $\mu$ calculados por el modelo Baranyi y Roberts a distintas temperaturas de almacenamiento. Con el fin de describir de manera sencilla el efecto de la temperatura de almacenamiento sobre el crecimiento de Salmonella spp en queso costeño, se utilizó una ecuación polinómica. Considerando las condiciones de abuso de temperatura a los cuales son sometidos los quesos costeños en la región Caribe colombiana, las cinéticas de crecimiento de 
Salmonella spp fueron desarrolladas en un rango de temperaturas $\left(8-21^{\circ} \mathrm{C}\right)$, donde el modelo pueda ser útil para predecir el comportamiento del microorganismo. Con el fin de verificar el desempeño del modelo construido, es necesario llevar a cabo una validación del modelo. Por tanto, algunos índices como Af y Bf han sido propuestos por diversos autores (Baranyi et al., 1999) para realizar validaciones en modelos polinómicos. Af es la suma de diferencias absolutas entre los valores predichos y observados de un parámetro determinado en el modelo secundario. Bf representa la desviación relativa entre los valores predichos y observados y es un índice utilizado para determinar si el modelo predice por encima o por debajo el crecimiento microbiano (Dalgaard y Jorgensen, 1998).

Valores de $\mathrm{Bf}$ reportados en la literatura van de 0,250 encontrado en un modelo de raíz cuadrada dependiente de la temperatura (Sant'Ana et al., 2012) hasta 3,190 en un tipo de ecuación de Gompertz propuesta por Park et al., (2007). Sin embargo, es importante aclarar que valores de Bf por fuera del rango 0,7 hasta 1,5 son considerados inconvenientes para predecir crecimiento microbiano; por lo que, una concordancia perfecta entre las predicciones y las observaciones indica valores de Af y Bf iguales a 1.0 (Choi et al., 2019; Ross, 1999). El último índice estadístico propuesto para validar modelos predictivos es el ECM, que es una comparación entre los valores observados en el experimento y aquellos valores calculados por el modelo en la misma temperatura; entre más cercano se encuentre este valor a cero indica que el modelo es más aceptable (Baranyi et al., 1996). En este estudio se obtuvieron valores ECM de 0,001; el cual es menor al reportado por Tirloni et al., (2021) quienes obtuvieron valores entre 0,994 y 0,991 después de modelar los valores de $\mu_{\max }$ en función de la temperatura usando la ecuación de Rosso et al., (1993).

El resultado del proceso de validación del modelo secundario construido para predecir el crecimiento de Salmonella spp se presenta en la tabla 3, en donde se aprecian valores de Af, Bf y ECM de 1,112;0,898 y 0,001 respectivamente. Estos resultados son similares a los publicados por $Y u$ et al., (2020), quienes reportaron valores de Af $(1,06-1,13) \mathrm{Bf}(0,91-1,00)$ y $\operatorname{ECM}(0,00-0,68)$ durante la validación de un modelo secundario para predecir el crecimiento de $S$. aureus en carne bovina cruda en diversas condiciones de envasado. Los resultados sobre la validación del modelo secundario construido son aceptables para describir el crecimiento de Salmonella spp en queso costeño, teniendo en cuenta que la mayoría de los modelos validados y disponibles para Salmonella spp. no han sido apropiados debido a que subestimaron el crecimiento de este patógeno (Sant'Ana et al., 2012). También es importante destacar que los valores predichos por los modelos difieren a menudo de los valores observados debido a las diferencias en las matrices alimentarias ( $\mathrm{pH}$, contenido de humedad, concentración de sal y compuestos incorporados) Tirloni et al., (2021). Por este motivo, se determinaron algunos parámetros fisicoquímicos del queso que pueden afectar el crecimiento microbiano.

Los valores de pH estuvieron entre 6,83 y 5,53; los valores de sal fueron de 2,78 hasta 3,18\% apreciándose un leve incremento al finalizar la cinética de crecimiento. Se observó una leve disminución de las grasas en los quesos, que puede ser atribuible al metabolismo de los microorganismos. Aunque esta leve disminución puede ser consecuencia por el corto tiempo de muestreo. La grasa es uno de los parámetros de mayor interés; ya que contribuyen con el sabor del producto final. Los mayores valores de grasa se encontraron al iniciar la cinética de crecimiento en $28,31 \pm 0,37 \%$; mientras que al final de la cinética de crecimiento se obtuvieron valores de $23,57 \%$. Similar comportamiento fue obtenido en los valores de proteína; los cuales estuvieron entre 25,33 y $18,94 \%$. Sin embargo, es importante mencionar que las variaciones en los análisis fisicoquímicos también pueden ser causados por la calidad de la leche empleada para elaborar los quesos, así como de la microbiota presente en el producto final. Hay muchos modelos disponibles para predecir el crecimiento de Salmonella spp, la mayoría considerando caldos microbiológicos y productos cárnicos (Pin et al., 2011). En este estudio se utilizaron muestras de queso costeño en lugar de los tradicionales medios de cultivo, por tanto, el modelo desarrollado puede ser más consistentes para uso práctico en la industria quesera colombiana.

Tabla 3. Validación matemática del modelo secundario desarrollado para describir el comportamiento de Salmonella spp en queso costeño

\begin{tabular}{|c|c|c|c|}
\hline Ecuación polinómica & $A f$ & $B f$ & $E C M$ \\
\hline $\log (\mu)=0,0001 \mathrm{x}^{2}-0,00186 \mathrm{x}+0,0146$ & 1,112 & 0,898 & 0,001 \\
\hline
\end{tabular}

\section{CONCLUSIONES}

La velocidad de crecimiento y la concentración microbiana final mostraron ser dependientes de los cambios de temperatura. Debido a los valores de AF, Bf y ECM obtenidos, se puede inferir que el modelo construido puede predecir de manera correcta el crecimiento de Salmonella spp en queso costeño en condiciones de refrigeración y abuso de temperatura; por tanto, podría ser utilizado en la evaluación cuantitativa de riesgos para determinar el crecimiento de Salmonella spp en queso costeño. 


\section{MATERIAL SUPLEMENTARIO}

El desarrollo más completo del modelo, así como las condiciones específicas de crecimiento de Salmonella spp puede ser obtenido del autor(a) de correspondencia rgonzalezc1@unicartagena.edu.co

\section{REFERENCIAS}

Aćai, P., Valík, I., Medvedová, A., y Studenićová, A., Staphylococcus aureus in unripened ewes' lump cheese. Part 1: Exposure assessment after first 24 h of fermentation, J. Food Nutrition Res., 53(2), 143-151 (2014)

Baranyi, J., y Roberts, T.A., A dynamic approach to predicting bacterial growth in food, https://doi.org/10.1016/01681605(94)90157-0, Int. J. Food Microbiology, 23(3-4), 277-294 (1994)

Baranyi, J., Ross, T., McMeekin, T. A., y Roberts, T. A., Effects of parameterization on the performance of empirical models used in "predictive microbiology", https://doi.org/10.1006/fmic.1996.0011, Food Microbiol., 13(1), 83-91 (1996)

Baranyi, J., Pin, C., y Ross, T., Validating and comparing predictive models, https://doi.org/10.1016/S01681605(99)00035-5, Int. J. Food Microbiol., 48(3), 159-166 (1999)

Choi, W., Son, N., y otros seis autores, Predictive model of Staphylococcus aureus growth on egg products, DOI: 10.1007/s10068-018-0529-4, Food Science and Biotechnol., 28(1), 913-922 (2019)

Dalgaard, P., y Jorgensen, L.V., Predicted and observed growth of Listeria monocytogenes in seafood challenge tests and in naturally contaminated cold smoked salmon, DOI: 10.1016/s0168-1605(98)00019-1, Int. J. Food Microbiology, 40 (1-2), 105-115 (1998)

Gutiérrez, C., Quintero, R., Burbano, I., y Simancas, R., Modelo de quesería artesanal bajo un signo distintivo en el Caribe colombiano: caso Atlántico, https://doi.org/10.22507/rli.v14n1a6, Rev L de Invest., 14(1), 72-83 (2017)

Herrero, A., y Olsen, J., Salmonella typhimurium metabolism affects virulence in the host - a mini-review, https://doi.org/10.1016/j.fm.2017.04.016, Food Microbiology, 71, 98-110 (2018)

Juneja, V., Valenzuela, M., y otros cuatro autores, Modeling the effect of temperature on growth of Salmonella in chicken, https://doi.org/10.1016/j. fm.2006.08.004, Food Microbiology, 24(4), 328-335 (2007)

Jung, J., y Schaffner, D., Modeling the survival of Salmonella on whole cucumbers as a function of temperature and relative humidity, https://doi.org/10.1016/j.fm.2021.103840, Food Microbiology, 100, 103840 (2021)

Kipper, D., Hellfeldt, R., y otros cinco autores, Salmonella serotype assignment by sequencing analysis of intergenic regions of ribosomal RNA operons, https://doi.org/10.3382/ps/pez285, Poultry Science, 98(11), 5989-5998 (2019)

Lee, Y., Jung, B., y otros cuatro autores, Predictive model for the growth kinetics of Listeria monocytogenes in raw pork meat as a function of temperature, https://doi.org/10.1016/j.foodcont.2014.03.024, Food Control, 44, 16-21 (2014)

Leong, W., Geier, R., y otros cuatro autores, Growth of Listeria monocytogenes, Samonella spp., Escherichia coli O157:H7 and Staphylococcus aureus on cheese during extended storage at $25^{\circ} \mathrm{C}$, DOI: $10.4315 / 0362-028 X . J F P-14-$ 047, J. Food Prot., 77(8), 1275-1288 (2014)

Milkievicz, T., Badia, V., y otros cinco autores, Development of a general model to describe Salmonella spp. growth in chicken meat subjected to different temperature profiles, https://doi.org/10.1016/j.foodcont.2020.107151, Food Control, $112,107151,(2020)$

Milkievicz, T., Badia, V., y otros cinco autores, Modeling Salmonella spp. inactivation in chicken meat subjected to isothermal and non-isothermal temperature profiles, https://doi.org/10.1016/j.ijfoodmicro.2021.109110, Int J. Food Microbiology, 344, 109110 (2021)

Oliver, S., Jayarao, B., y Almeida, R., Foodborne pathogens in milk and the dairy farm environment: Food safety and public health implications, DOI: 10.1089/fpd.2005.2.115, Foodborne Pathog Dis., 2(2), 115-29 (2005)

Orosco, G., Vieira, T., Cardoso, M., y de Freitas Costa, E., Application of a predictive microbiological model for estimation of Salmonella behavior throughout the manufacturing process of salami in environmental conditions of smallscale Brazilian manufacturers, https://doi.org/10.1016/j.mran.2021.100177, Microbial Risk Analysis, en prensa, 100177 (2021)

Park, S., Seo, K., y Ha, S., A response surface model based on absorbance data for the growth rates of Salmonella enterica serovar Typhimurium as a function of temperature, $\mathrm{NaCl}$, and pH, J. Molecular Biotechnol., 17(4), 644-649 (2007)

Park, J., Kang, S., y otros seis autores, A dynamic predictive model for the growth of Salmonella spp. and Staphylococcus aureus in fresh egg yolk and scenario-based risk estimation, https://doi.org/10.1016/j.foodcont.2020.107421, Food Control, 118, 107421 (2020)

Pin, C., Avendaño, G., y otros seis autores, Modelling Salmonella concentration through the pork supply chain by considering growth and survival in fluctuating conditions of temperature, $\mathrm{pH}$ and aw, https://doi.org/10.1016/j.ijfoodmicro.2010.09.025, Int. J. Food Microbiology, 145 (1), S96-S102 (2011)

Ross, T., Predictive food microbiology models in the meat industry, ISBN 0958582513, 7(1), 114-120 (1999) 
Rosso, L., Lobry, J., y Flandrois, J., An unexpected correlation between cardinal temperatures of microbial growth highlighted by a new model, DOI: 10.1006/jtbi.1993.1099, J. Theor. Biol., 162(4), 447-463 (1993)

Sant'Ana, A., Franco, B., y Schaffner, D., Modeling the growth rate and lag time of different strains of Salmonella enterica and Listeria monocytogenes in ready-to-eat lettuce, https://doi.org/10.1016/j.fm.2011.11.003, Food Microbiology, 30(1), 267-272 (2012)

Sannigrahi, S., Arumugasamy, S. K., Mathiyarasu, J., y Suthindhiran, K., Magnetosome-anti-Salmonella antibody complex based biosensor for the detection of Salmonella typhimurium, https://doi.org/10.1016/j.msec.2020.111071, Mater Sci Eng C Mater Biol Appl., 114, 111071 (2020)

Skandamis, P., y Jeanson, S., Colonial vs. planktonic type of growth: mathematical modelling of microbial dynamics on surfaces and in liquid, semiliquid and solid foods, https://doi.org/10.3389/fmicb.2015.01178, Front. Microbiol., 6(1), 11-78 (2015)

Tarlak, F., Johannessen, G., y otros cuatro autores, Modelling of the behaviour of Salmonella enterica serovar reading on commercial fresh-cut iceberg lettuce stored at different temperatures, https://doi.org/10.3390/foods9070946, Foods, 9(7), 946 (2020)

Tirloni, E., Stella, S., y otros tres autores, Predicting growth of Listeria monocytogenes in fresh ricotta, https://doi.org/10.1016/j.fm.2018.10.004, Food Microbiol., 78, 123-133 (2019)

Tirloni, E., Stella, S., Bernardi, C., y Rosshaug, P., A new predictive model for the description of the growth of Salmonella spp. in Italian fresh ricotta cheese, https://doi.org/10.1016/j.Iwt.2021.111163, Food Science Technol., 143, 111-163 (2021)

Yu, H., Song Y., y otros cinco autores, Predictive model of growth kinetics for Staphylococcus aureus in raw beef under various packaging systems, doi: 10.1016/j.meatsci.2020.108108, Meat Sci., 165,108108 (2020)

Whiting, R., Microbial modeling in foods, https://doi.org/10.1080/10408399509527711, Crit. Rev. Food Sci. Nutr. 35(6), 464-494 (1995) 
